\title{
Detection of cancer-associated glycobiomarkers using lectin-based biosensors
}

\author{
M. Luísa S. Silva \\ Centre of Chemical Research, Autonomous University of Hidalgo State, México \\ mluisasilva@portugalmail.pt
}

\begin{abstract}
- Introduction
Lectin biosensors are attractive devices for the detection of cancer-associated glycobiomarkers in serum since they combine the advantageous aspects of biosensors (portability, easy use in point-of-care analysis, low sample requirement) with the high selectivity of lectin biorecognition. This work presents three lectin-based impedimetric biosensors for the selective detection of specific aberrant cancer-associated O-glycans, namely STn, Tn and T antigens, which are wellestablished pan-carcinoma biomarkers. For these three biosensors, Sambucus nigra agglutinin (SNA), Vicia villosa agglutinin (VVA) and Arachis hypogeae agglutinin (PNA) were used as biorecognition elements, with specificity for STn, Tn and T antigens, respectively. The binding event between each lectin and the corresponding aberrant $\mathrm{O}$-glycan was monitored by electrochemical impedance spectroscopy, measuring the increase in the biosensor's impedance after incubating the samples. The increase in impedance was related to the lectin-glycan complex formation [1-3].
\end{abstract}

- Biosensor construction

\begin{tabular}{|c|c|c|}
$\begin{array}{c}\text { cancer-associated } \\
\text { truncated O-glycan }\end{array}$ & $\begin{array}{c}\text { lectin immobilized } \\
\text { on the biosensor }\end{array}$ & $\begin{array}{c}\text { model glycoprotein used to } \\
\text { monitor complex formation }\end{array}$ \\
\hline STn & SNA & $\begin{array}{c}\text { bovine submaxillary mucin; } \\
\text { human transferrin }\end{array}$ \\
\hline Tn & VVA & $\begin{array}{c}\text { asialofetuin; } \\
\text { asialo-bovine submaxillary } \\
\text { mucin }\end{array}$ \\
\hline T & PNA & asialofetuin \\
\hline
\end{tabular}
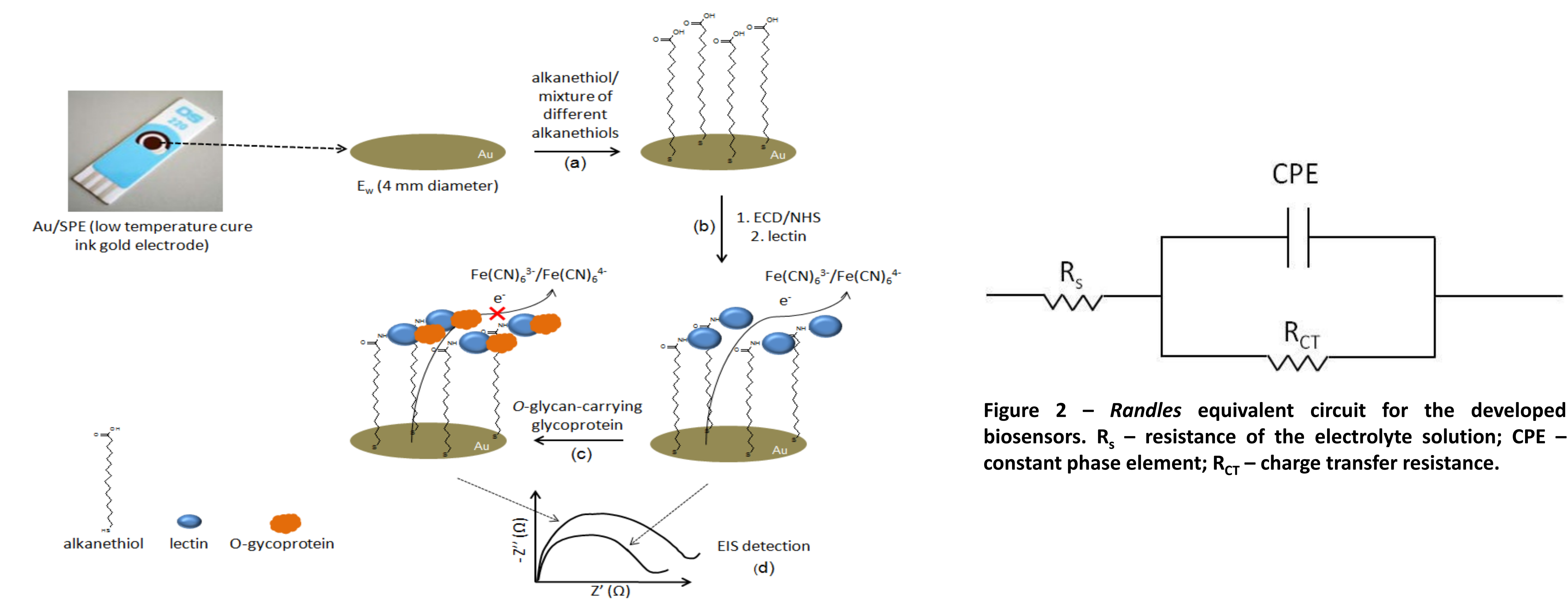

Figure 2 - Randles equivalent circuit for the developed biosensors. $R_{s}$ - resistance of the electrolyte solution; $\mathrm{CPE}$ constant phase element; $R_{\mathrm{CT}}-$ charge transfer resistance.

Figure 1 - Schematic diagram describing the construction of each lectin biosensor and detection of aberrant $O$-glycans by EIS: (a) alkanethiol/mixed alkanethiols self-assembled monolayer is formed via incubation of screen-printed binding with the lectin; (c) the truncated $O$-slycan present in glycoproteins is captured based on the affinity of the lectin to the referred structure; (d) the formation of the complex lectin-truncated $O$-glycan is monitored by the increase in the electrode impedance (by electrochemical impedance spectroscopy).

\section{- Selectivity}

SNA biosensor
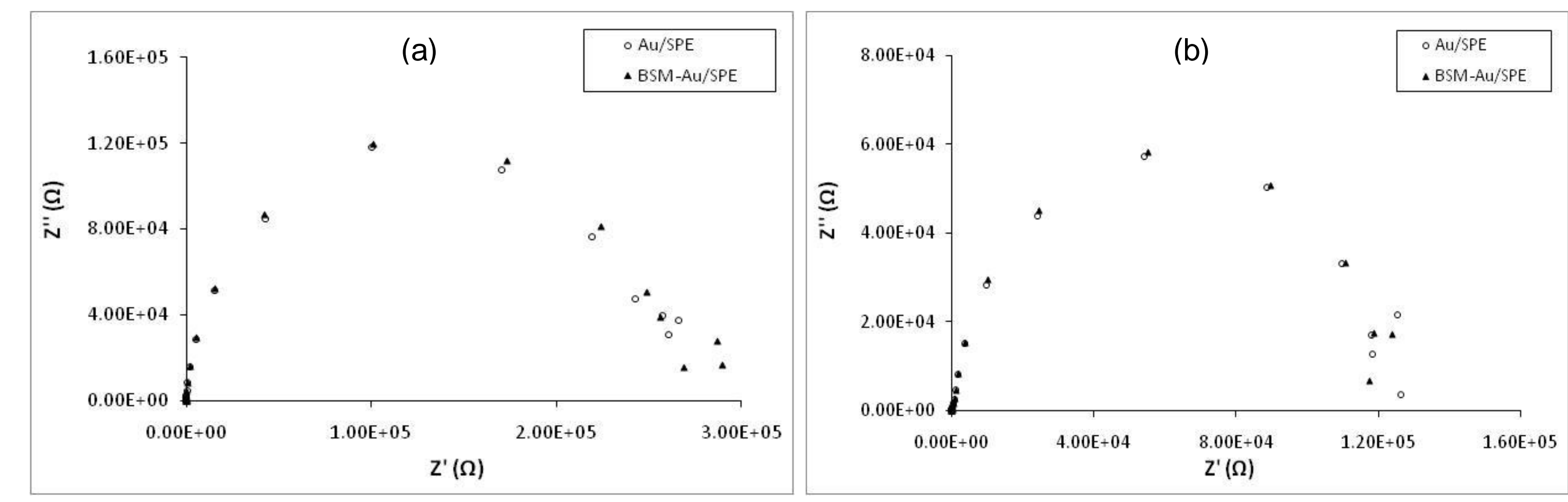

Figures 3 and 4 - Nyquist plots obtained before and after incubating the blank biosensor (with no lectin) with BSM solutions (a) $0.01 \mu \mathrm{g} \mathrm{ml} l^{-1}$ and (b) $1.0 \mu \mathrm{g} \mathrm{ml} l^{-1}$, for $5 \mathrm{~min}$ at room temperature.
VVA biosensor

PNA biosensor

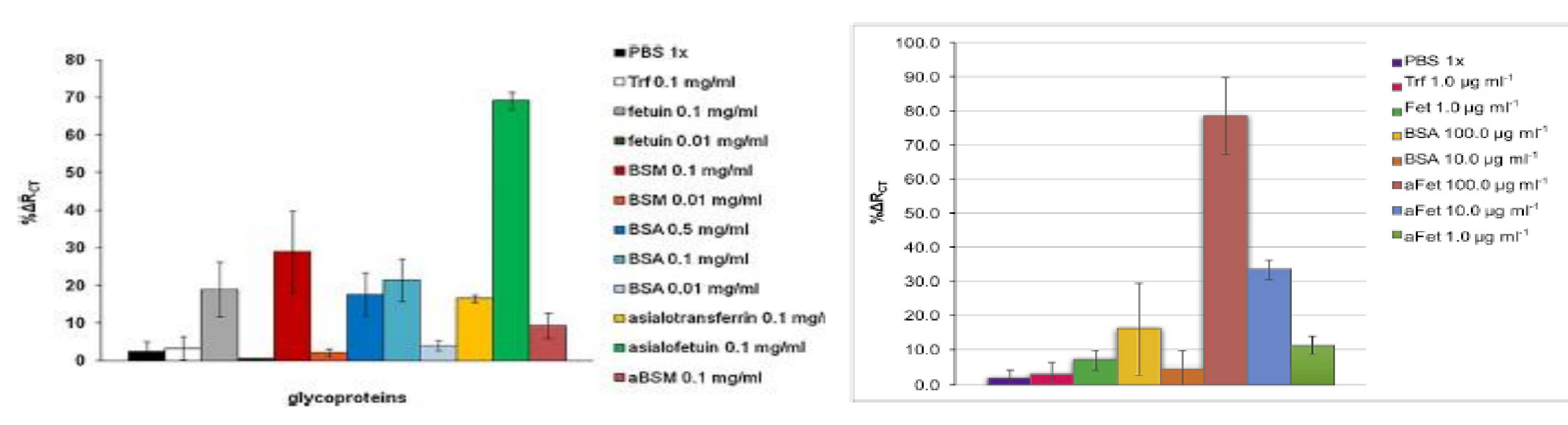

Figures 5 and 6 - Response for several glycoprotein solutions, incubated for 10
duplicate measurements with two independent biosensors for each solution.
- Sample analysis

Figure 7 - Graphical representation of the first two scores of a PCA performed on the impedimetric data from sample analysis using the SNA biosensor. Each point represents an individual analysis of a sample; (a) - breast malignant tumour (c and entoneal located different cancer samples, (d) -cervicaluterine carcinoma.

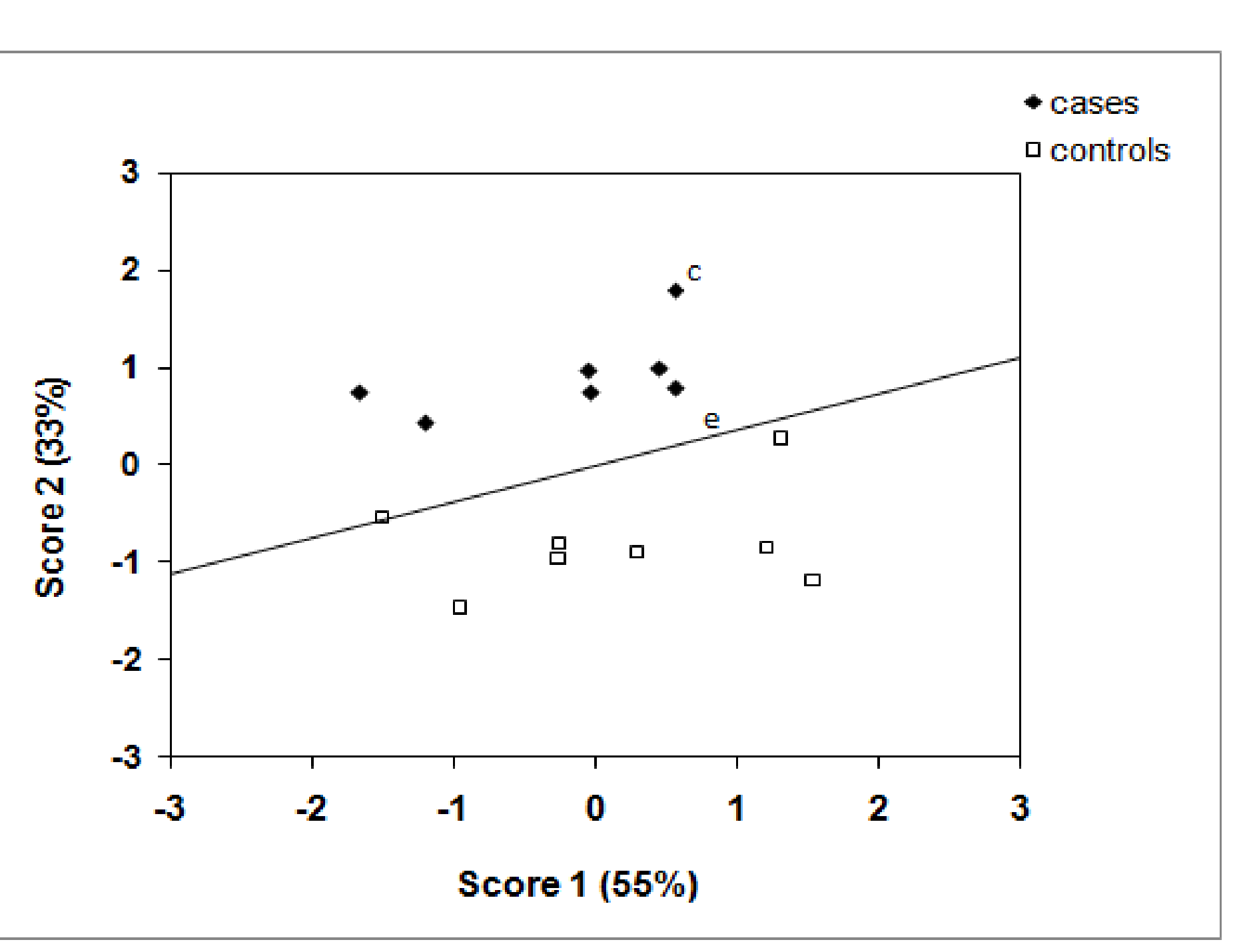

Figure 8 - Results obtained in sample analysis for VVA and PNA biosensors. Each sample pool refers to a type of carcinoma. Ctrl represents a pool of samples from healthy deviations of duplicate mearure standard two independent biosensors for each solution.

\section{- Conclusions}

1. All biosensors were constructed following the same general procedure, demonstrating its high versatility.

2. The three biosensors correctly discriminated samples from healthy donors and from cancer patients with different carcinomas, showing high selectivity towards the antigens STn, Tn and T.

3. Using the three lectin biosensors in the analysis of the same sample allowed to characterize the glycosylation profiles of glycoproteins in the diverse types of carcinomas.

References: [1] M. Luísa S. Silva, Evelin Gutiérrez, José A. Rodríguez, Catarina Gomes, Leonor David. Biosens. Bioelectron. 57 (2014) 254-261. [2] M. Luísa S. Silva, María G. H. Rangel. Sens. Actuators B 252 (2017) 777-784. [3] María. G. H. Rangel, M. Luísa S. Silva. Biosens. Bioelectron. 141 (2019) 111401. 